\title{
Familial cramp due to potassium-aggravated myotonia
}

\author{
Richard W Orrell, Karin Jurkat-Rott, Frank Lehmann-Horn, Russell J M Lane
}

Department of

Neuromuscular

Diseases, Division of

Neuroscience and

Psychological

Medicine, Imperial

College School of

Medicine, Charing

Cross Hospital,

London, UK

$\mathrm{R}$ W Orrell

R J M Lane

Department of Applied Physiology, University

of Ulm, Germany

K Jurkat-Rott

F Lehmann-Horn

Correspondence to: Dr Richard W Orrell, Department of Clinical Neurosciences, Royal Free and University College Medical School of University College London, Rowland Hill Street, London NW3 2PF, UK.

Telephone 00441710500 ; fax 00441714311577 ; email rfns0010@rfhsm.ac.uk

Received 8 December 1997 and in revised form

25 February 1998

Accepted 6 April 1998

\begin{abstract}
Clinical, electrophysiological, and molecular genetic features were investigated in two patients from a family a with dominantly inherited myotonic disease, characterised by painful cramps, stiffness without weakness, fluctuation of symptoms, and cold sensitivity. A reduction in amplitude of the compound muscle action potential was demonstrated on cooling and administration of potassium, although no clinical exacerbation was seen. A heterozygote mutation Val1589Met was identified in the $\alpha$-subunit of the skeletal muscle sodium channel gene in both patients, consistent with the diagnosis of potassium-aggravated myotonia. The phenotype in this family is much milder than that previously described in another family with a mutation at this site.

(F Neurol Neurosurg Psychiatry 1998;65:569-572)
\end{abstract}

Keywords: cramp; sodium channel disease; potassiumaggravated myotonia

Myotonia is a stiffness of the muscles, with inability to relax after contraction, induced by mechanical or electrical excitation. It is due to hyperexcitability of the muscle fibres, resulting from changes in ion flux homeostasis at the muscle sarcolemma, and is a manifestation of various primary muscle diseases including myotonic dystrophy, hyperkalaemic periodic paralysis, myotonia congenita, paramyotonia congenita, and potassium-aggravated myotonia $^{12}$; muscle stiffness of neurogenic origin is termed neuromyotonia. Myotonia may be described in several ways by patients, including a complaint of recurrent "cramps".

Molecular diagnosis has allowed the pathogenetic classification of these phenotypes, in particular the trinucleotide repeat expansion in the DMPK (myotonin-protein kinase) gene in myotonic dystrophy, the muscle chloride channel mutations of myotonia congenita, and the muscle sodium channel mutations of hyperkalaemic periodic paralysis, paramyotonia congenita, and potassium-aggravated myotonia. ${ }^{2-4}$ Potassium-aggravated myotonia is the most recently defined entitiy, ${ }^{56}$ having initially been termed sodium channel myotonia, ${ }^{7-9}$ and includes the conditions acetazolamide responsive myotonia, ${ }^{10}$ myotonia fluctuans, ${ }^{11-13}$ and myotonia permanens, ${ }^{8}$ all associated with mutations in the $\alpha$-subunit of the sodium channel gene (SCN4A). We describe a family with a much milder phenotype of potassium-aggravated myotonia than previously reported for a mutation at this site.

\section{Case report}

A 20 year old university student (IV.11, fig 1) presented with difficulty in prolonged writing, especially during examinations, and symptoms of sensory disturbance in the forearm, characterised by painful pins and needles. These symptoms had first developed several months earlier. She had experienced cramps in the toes, fingers, and eyelids, especially when tired or cold, throughout her life. Physical examination disclosed very mild orbicularis oculi myotonia, and mild percussion myotonia of the thenar eminence. There was no grip myotonia, or weakness, or wasting of the muscles.

Her 54 year old mother (III.11) had a long history of cramp-like sensations in the muscles

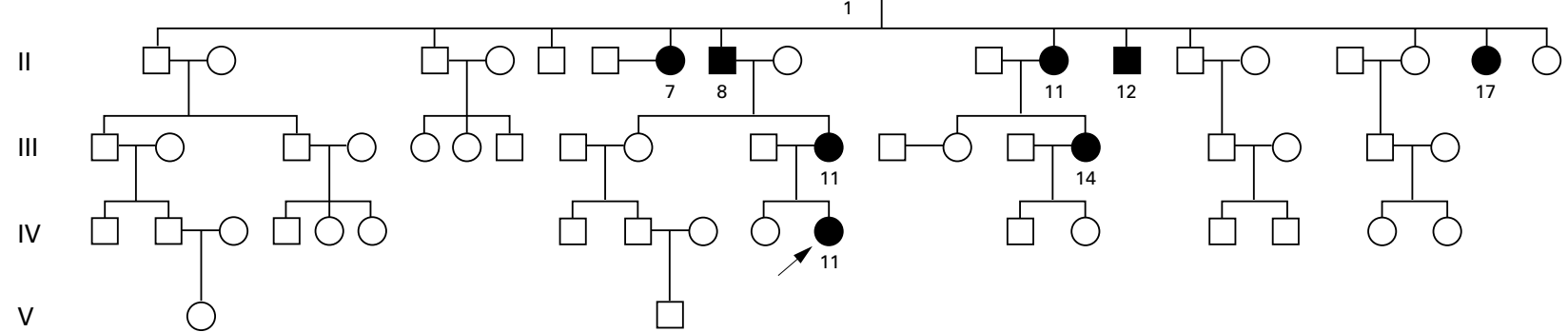

Figure 1 Pedigree of family with autosomal dominant inheritance of potassium-aggravated myotonia. Squares represent males, circles represent females, filled symbols are affected members, arrow indicates proband. 


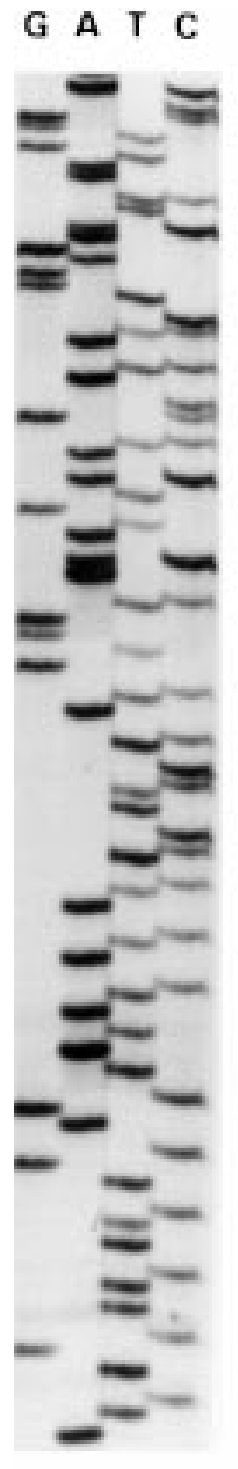

G A T C
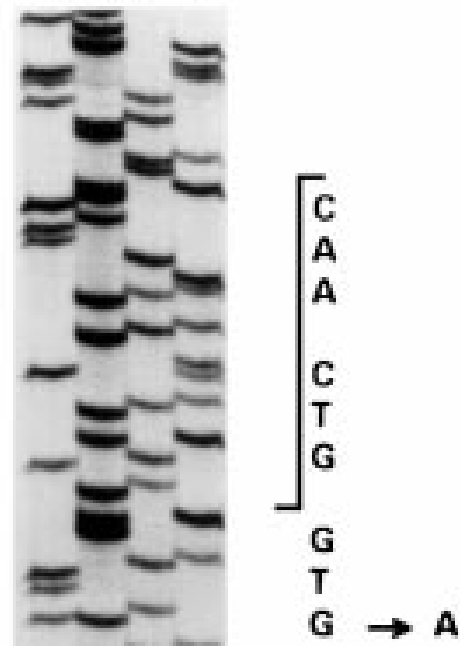

Asn

Val

Val $\rightarrow$ Met

$7^{\mathrm{T}}$

A

lle

C

T

C

$5^{\prime}$

Leu

Normal

\section{Val 1589 Met}

Figure 2 Illustration of mutation Val1589Met in SCN4A. G=guanine, $A=$ adenine, $T=$ thymine, $C=$ cytosine. The mutation is a heterozygote, and both normal and mutant sequences are present in the affected member, the additional $A$ band leading to the translation of amino acid methionine (Met) rather than valine (Val).

of the legs (especially calf and toes) and noted difficulty with opening her eyes after tight closure. She also noticed a sensation of muscle spasm in the eyelids in cold weather and when she was tired. She had noticed difficulty in alternating between extremes of gaze. The symptoms could be more diffuse when exerting strenuously or in the cold, and when swimming. She had never experienced episodes of weakness or paralysis. She had experienced symptoms since birth, apparently being born with one arm in a fixed flexion posture. The symptoms had eased a little with age. On examination she had more marked orbicularis oculi myotonia than her daughter, and percussion myotonia of the thenar eminence, with grip myotonia. The grip myotonia increased in the first two to three movements and then eased, and the orbicularis oculi myotonia also increased initially and then eased on repetitive action. There was no weakness or wasting of the limbs. General investigation including serum sodium, potassium, and ECG were normal. Serum creatine kinase was mildly raised at 402 U/1 (normal 0-150).

The mother had accepted her symptoms as normal as other members in four generations of her family (of white origin) had also experienced similar symptoms, which had not previously caused appreciable disability (fig 1). Family member III.14 experienced similar symptoms in the hands and feet, with difficulty opening her hands in the cold. In cold weather she experienced transient diplopia on rapid eye movement. She also experienced cramps "in the diaphragm". Other relatives with similar symptoms were I.1, II.6, II.8, II.10, II.12, and II.17. None had sought medical advice for their symptoms.

\section{Methods}

ELECTROPHYSIOLOGICAL INVESTIGATION

Nerve conduction studies and EMG were performed in conventional manner. A short exercise test ${ }^{14}{ }^{15}$ was performed by the proband (IV.11) and her mother (III.11). This involved recording the abductor digiti minimi compound muscle action potential (CMAP) after stimulation of the ulnar nerve at the wrist. Disc surface electrodes $(5 \mathrm{~mm})$ were taped to the skin over the abductor digiti minimi (the active electrode over the hypothenar eminence and the reference over the proximal part of the fifth finger), and the ulnar nerve was stimulated with surface electrodes applied at the wrist. The electrodes were firmly secured in position and the finger, arm, and hand were immobilised using an arm board. Supramaximal stimulation of the ulnar nerve was performed (ambient temperature $24^{\circ} \mathrm{C}$, skin temperature $32^{\circ} \mathrm{C}$ ) with the peak to peak CMAP amplitude recorded at 30 second intervals for 2 minutes to establish a baseline. The patient then contracted ADM isometrically for 20 seconds. The CMAP amplitude was recorded every 10 seconds after exercise until no further decrease in amplitude was seen. The percentage decrement of CMAP amplitude after exercise was calculated when present. The limb was then cooled by immersion, with electrodes and splint in situ, within a polythene bag in a bucket of iced water. The skin surface temperature was reduced to $16^{\circ} \mathrm{C}$, and the limb removed and allowed to warm to $25^{\circ} \mathrm{C}$ before repeating the exercise test. The tests were repeated in a normal volunteer with no evidence of neuromuscular disease.

\section{MOLECULAR GENETICS}

DNA was extracted from whole blood in EDTA using standard methods. Exons 1-24 of SCN4A, the gene encoding the $\alpha$-subunit of the sodium channel protein, were amplified by polymerase chain reaction (PCR), and the products examined for mutations by single stranded conformation polymorphism analysis (SSCP), and by direct PCR sequencing, as previously described. ${ }^{16}$ In figure 2 , the primers used were 5' CCT CCT CCT CTT CCT GGT CAT and 5' GGG CTC GCT GCT 
Results of the short exercise test performed at room temperature and after cooling of the arm to $16^{\circ} \mathrm{C}$, in the proband (IV.11), her mother (III.11), and a normal unaffected person, as described in the text

\begin{tabular}{|c|c|c|c|c|c|c|}
\hline & \multicolumn{2}{|c|}{ Proband } & \multicolumn{2}{|c|}{ Mother } & \multicolumn{2}{|c|}{ Normal person } \\
\hline & $\begin{array}{l}\text { Room } \\
\text { temp }\end{array}$ & Cooled & $\begin{array}{l}\text { Room } \\
\text { temp }\end{array}$ & Cooled & $\begin{array}{l}\text { Room } \\
\text { temp }\end{array}$ & Cooled \\
\hline Minimum CMAP amplitude before exercise $(\mathrm{mV})$ & 6.1 & 5.8 & 15.8 & 15.3 & 13.0 & 13.5 \\
\hline Minimum CMAP amplitude after exercise (mV) & 6.0 & 4.8 & 16.3 & 11.7 & 13.1 & 14.6 \\
\hline Decrement of CMAP after exercise (\%) & 2.0 & 17.0 & 0.0 & 24.0 & 0.0 & 0.0 \\
\hline Maximum CMAP amplitude after exercise (mV) & 6.3 & 5.4 & 17.7 & 15.8 & 14.4 & 15.9 \\
\hline
\end{tabular}

$\mathrm{CMAP}=$ compound muscle action potential.

CTC CTC TGT (biotinylated); the products were purified with Dynal beads, sequenced with Sequenase in the presence of $\left[{ }^{35} \mathrm{~S}\right] \mathrm{dATP} \alpha \mathrm{S}$, separated on a $6 \%$ polyacrylamide gel, and exposed to autoradiographic film, as described. ${ }^{17}$

\section{Results}

ELECTROPHYSIOLOGICAL INVESTIGATION In the proband (IV.11) there was no abnormality of motor or sensory conduction. EMG of the right abductor digiti minimi showed fibrillations and positive sharp waves with profuse myotonic discharges at rest. The interference pattern was normal, with normal motor units. After cooling the hand to $16^{\circ} \mathrm{C}$ the spontaneous activity and myotonic discharges were reduced but not abolished. Similar EMG findings were present in her mother (III.11), with abolition of fibrillations and positive sharp waves, but persistence of moderate myotonic discharges on cooling. There was a reduction in CMAP amplitude on cooling the limb (table). There was no significant clinical weakness or increase in myotonia in the limb on cooling. On a subsequent occasion, the mother (III.11) was given $80 \mathrm{mmol} \mathrm{KCl}$ (without glucose) orally. ${ }^{14}{ }^{16} \mathrm{By}$ 30 minutes she experienced a sensation of muscle spasm around the eyes, and increased stiffness on walking, similar to that normally precipitated by cold. The symptoms persisted for around 3 hours but were not appreciably disabling, and no clinical change was seen. Serum potassium increased from $3.5 \mathrm{mmol} / 1$ at baseline to $5.0 \mathrm{mmol} / \mathrm{l}$ at 45 minutes. The short exercise test was repeated. On this occasion, there was no decrement in CMAP amplitude before potassium administration, and no decrement after potassium administration at room temperature, but a $10 \%$ decrease $(14.1 \mathrm{mV}$ maximum, $12.7 \mathrm{mV}$ minimum) after cooling.

\section{MOLECULAR GENETICS}

The mutation of guanine to adenine at base 4765 , with substitution of methionine for valine at codon 1589, was identified in exon 24 of SCN4A (fig 2), in both III.11 and IV.11. No other mutation of SCN4A was identified.

\section{Discussion}

Mutations in genes encoding ion channels have been identified as the cause of a wide range of diseases including myotonias and periodic paralyses, episodic ataxias, seizures, long QT syndrome (in the heart), hypertension (Liddle syndrome), cystic fibrosis, startle disease, and nephrogenic diabetes insipidus. ${ }^{18}$ The human muscle sodium channel is a voltage dependent channel, composed of a major $\alpha$-subunit and a $\beta$-subunit. The $\alpha$-subunit gene (SCN4A) is on chromosome 17q23.1-25.3 and comprises 24 exons. It encodes 1836 amino acids, forming a $260 \mathrm{kDa}$ glycoprotein. The $\alpha$-subunit consists of four homologous domains (DI-IV) each containing six transmembrane segments $\left(\right.$ S1-6). ${ }^{419}$ Around 20 different mutations of the human muscle sodium channel have now been identified, and the resulting phenotypes (the sodium channel diseases) can be classified as four groups: hyperkalaemic periodic paralysis, normokalaemic periodic paralysis, paramyotonia congenita, and potassium-aggravated myotonia. $^{2}{ }^{4}$ 20-22 $^{2}$ These diseases show autosomal dominant inheritance, with variable penetrance, and de novo mutations may occur. Hyperkalaemic and normokalaemic periodic paralysis are associated with episodes of paralysis and in some cases may be associated with clinical or electrophysiological myotonia, especially at the beginning of an attack. ${ }^{2}$ Paramyotonia congenita manifests with paradoxical myotonia (increasing with exercise, as opposed to the normal "warm up" improvement with exercise in classic myotonia), which is exacerbated by cold, and shows predilection for face, neck, and long muscles of the hands, together with weakness after protracted exercise and exposure to cold. ${ }^{2}$ Some patients may also have episodes of muscle weakness with hyperkalaemia. Potassium-aggravated myotonia may be confused clinically with myotonia congenita. ${ }^{2410}$ Weakness is not a feature of this condition, but the stiffness may be exacerbated by exercise or cold.

The correlation of phenotype with SCN4A genotype is complex, and the same genotype seems responsible for varying phenotypes in some families. ${ }^{2123}$ The mutation we describe, causing potassium-aggravated myotonia, occurred in the S6 segment of repeat IV, adjacent to the mutation at 1592 which causes hyperkalemic periodic paralysis. ${ }^{40}$ Both mutations affect highly conserved amino acids close to the cytoplasmic surface, and induce an increase in the number of non-inactivating sodium channels. ${ }^{16}$ This region of the sodium channel seems to be important for fast inactivation, and may act as the acceptor of the inactivation gate according to the "ball and chain" model. ${ }^{24}$ The difference in phenotype between hyperkalaemic periodic paralysis and paramyotonia congenita, and potassium-aggravated myotonia (stiffness rather than weakness) may reflect the extent of sodium channel inactivation; a slight sustained depolarisation causing membrane hyperexcitability with resultant muscle stiff- 
ness, and a larger depolarisation causing membrane inexcitability with weakness or paralysis. ${ }^{162526}$ The short exercise test determines the excitability of the muscle membrane, and shows the sensitivity to cold and potassium even when there is no evident weakness. In the patient we tested with potassium load the response was mild, with no clinically apparent change, although altered excitability was detected on the short exercise test. A patient from the family reported by Heine et $a l^{16}$ with the same mutation (Val1589Met), was given the same dose of potassium $(80 \mathrm{mmol})$ and "the muscles became very stiff within 30 minutes ... and the patient was unable to rise or walk". The Val1589Met mutant sodium channel identified in our patients has been expressed and studied in human embryonic kidney (HEK293) cells, in which an instability of the inactivated state was shown. ${ }^{25}$ In view of the insignificant clinical response in our patient to oral potassium load, it is interesting that in these studies of the mutation in HEK293 cells, increasing the extracellular potassium concentration did not affect the current recorded. ${ }^{25}$

These patients also exhibit variability of symptoms from day to day, which has previously been described as myotonia fluctuans. ${ }^{13}$ An unusual feature is the occurrence of painful cramps, as was the presenting feature in our proband. Indeed, the first linkage of potassium-aggravated myotonia (or sodium channel myotonia) to the sodium channel locus was performed in patients originally thought to have an autosomal dominant form of myotonia congenita with painful myotonia and acetazolamide responsiveness. ${ }^{7}$ They also had prominent orbicularis oculi myotonia which was present in our patients. ${ }^{10}$ Symptomatic treatment of potassium-aggravated myotonia includes acetazolamide, and membrane stabilisers such as mexilitine and tocainide, but these were not tolerated or needed by our patients, who had mild symptoms.

1 Streib EW. Differential diagnosis of myotonic syndromes. AAEE minimonograph 27. Muscle Nerve 1987;10:603-15. 2 Rudel R, Lehmann-Horn F. Muscle sodium channel and chloride channel diseases. In: Lane RJM. Handbook of muscle disease. New York: Marcel-Dekker, 1996;339-53.

3 McClatchey AI, McKenna-Yasek D, Cros D, et al. Novel mutations in families with unusual and variable disorders of the skeletal muscle sodium channel. Nat Genet 1992;2:148-52.

4 Lehmann-Horn F, Rudel R. Hereditary non-dystrophic myotonias and periodic paralysis. Curr Opin Neurol 1995;8: 402-10.

5 Hoffman EP, Lehmann-Horn F, Rudel R. Over-excited or inactive: ion channels in muscle disease. Cell 1995;80:681-

6 Rudel R, Lehmann-Horn F. Workshop report. Paramyotonia, potassium-aggravated myotonias and periodic paralynia, potassium-aggravated myotonias

7 Ptacek LJ, Tawil R, Griggs RC, et al. Linkage of atypical myotonia congenita to a sodium channel locus. Neurology 1992;42:431-3.

8 Lerche H, Heine R, Pika U, et al. Human sodium channel myotonia: slowed channel inactivation due to substitutions for a glycine within the III-IV linker. F Physiol 1993;470:1322.

9 Ptacek LJ, Tawil R, Griggs RC, et al. Sodium channel mutations in acetazolamide-responsive myotonia congenita, paramyotonia congenita, and hyperkalemic periodic paralysis. Neurology 1994;44:1500-3.

10 Trudell RG, Kaiser KK, Griggs RC. Acetazolamideresponsive myotonia congenita. Neurology 1987;37:488-91.

11 Ricker K, Lehmann-Horn F, Moxley RT. Myotonia fluctuans. Arch Neurol 1990;47:268-72.

12 Lennox G, Purves A, Marsden D. Myotonia fluctuans. Arch Neurol 1992;49:1010-11.

13 Ricker K, Moxley RT, Heine R, et al. Myotonia fluctuans. A third type of muscle sodium channel disease. Arch Neurol 1994;51:1095-102.

14 Streib EW. Paramyotonia congenita: successful treatment with tocainide. Clinical and electrophysiological findings in seven patients. Muscle Nerve 1987;10:155-62.

15 Jackson CE, Barohn RJ, Ptacek LJ. Paramyotonia congenita: abnormal short exercise test, and improvement after mexiletine therapy. Muscle Nerve 1994;17:763-8.

16 Heine R, Pika U, Lehmann-Horn F. A novel SCN4A mutation causing myotonia aggravated by cold and potassium. Hum Mol Genet 1993;2:1349-53.

17 Orrell RW, Habgood JJ, Gardiner I, et al. Clinical and functional investigation of 10 missense mutations and a novel frameshift insertion mutation of the gene for copper-zinc superoxide dismutase in UK families with amyotrophic latsuperoxide dismutase in UK families with

18 Keating MT, Sanguinetti MC. Pathophysiology of ion channel mutations. Curr Opin Genet Dev 1996;6:326-33.

19 George AL, Komisarof J, Kallen RG, et al. Primary structure of the adult human skeletal muscle voltage-dependent sodium channel. Ann Neurol 1992;31:131-7.

20 Rojas CV, Wang J, Schwartz LS, et al. A Met-to-Val mutation in the skeletal muscle $\mathrm{Na}^{+}$channel $\alpha$-subunit in hyperkalaemic periodic paralysis. Nature 1991;354:387-9.

21 Rudel R, Ricker K, Lehmann-Horn F. Genotype-phenotype correlations in human skeletal muscle sodium channel disease. Arch Neurol 1993;50:1241-8.

22 Hudson AJ, Ebers GC, Bulman DE. The skeletal muscle sodium and chloride channel diseases. Brain 1995;118: 547-63.

23 Plassart E, Eymard B, Maurs L, et al. Paramyotonia congenita: genotype to phenotype correlations in two families and report of a new mutation in the sodium channel gene. 7 Neurol Sci 1996;142:126-33.

24 Caldwell JH, Schaller KL. Opening the gates on ion channel diseases. Nat Genet 1992;2:87-9.

25 Mitrovic N, George AL, Heine R, et al. $\mathrm{K}^{+}$-aggravated myotonia: destabilization of the inactivated state of the human muscle $\mathrm{Na}^{+}$channel by the V1589M mutation. $\mathcal{F}$ Physiol 1994;478:395-402.

26 Lerche H, Mitrovic N, Dubowitz V, et al. Paramyotonia congenita: the R1448 $\mathrm{P} \mathrm{Na}^{+}$channel mutation in adult human skeletal muscle. Ann Neurol 1996;39:599-608. 